\title{
Efecto de n-acetilglucosamina sobre plaquetas
}

\author{
Laura Pérez-Campos Mayoral', María del Socorro Pina-Canseco', \\ Eduardo Lorenzo Pérez-Campos ${ }^{1,2}$, María Teresa Hernández-Huerta ${ }^{2}$, \\ Edgar Zenteno-Galindo ${ }^{3}$, Ruth Martínez-Cruz ${ }^{1}$ \\ ${ }^{1}$ Centro de Investigación Facultad de Medicina, UNAM-UABJO \\ Universidad Autónoma "Benito Juárez" de Oaxaca \\ laurapcm@prodigy.net.mx, socopinal2@hotmail.com y perezcampos123@yahoo.es \\ ¿Unidad de Bioquímica e Inmunología, División de Estudios de Posgrado e Investigación \\ Instituto Tecnológico de Oaxaca, México marte-hh28@hotmail.com \\ ${ }^{3}$ Universidad Nacional Autónoma de México, México ezenteno@servidor.unam.m
}

\begin{abstract}
Resumen
El tratamiento de plaquetas con varios amino-azúcares, entre los que se encuentran: glucosamina, N-acetilglucosamina, ácido N-acetil neuraminico y otros, son capaces de inducir: cambios de forma, incremento en los niveles de calcio libre, exposición en la superficie de las plaquetas la fosfatidilserina, además de reducir la liberación de ATP y $\mathrm{PF}^{4}$ e inhibir la agregación plaquetaria. El objetivo de este trabajo fue evaluar el efecto de $\mathrm{N}$-acetilglucosamina sobre las plaquetas de Sujetos con Resistencia a la insulina, comparado con sujetos sanos. Las plaquetas $(80,000$ plaquetas $/ \mathrm{mL})$ de sujetos con resistencia a la insulina fueron lavadas e incubadas con $\mathrm{N}$-acetilglucosamina durante 10 minutos, y marcadas con los anticuerpos anti-PAC-1 y anti-CD62P acoplados a fluorocromos, y $\mathrm{Ca}_{2}+$ mediante la unión a Fluo-3. Nuestros resultados indican que $\mathrm{N}$-acetilglucosamina inhibe el calcio intraplaquetario inducido por trombina, la unión de los anticuerpos anti-GPIlb/IIla y anti-P-selectina.
\end{abstract}

Palabras clave: N-acetilglucosamina, activación plaquetaria

\section{Summary}

The treatment of platelets with various amino-sugars such as glucosamine, $\mathrm{N}$-acetylglucosamine, $\mathrm{N}$-acetyl neuraminic acid and others is capable of inducing: changes in shape, increased levels of free calcium, the exposure of phosphatidylserine on platelet surfaces, in addition to reducing the release of ATP and PF4 and inhibiting platelet aggregation. The objective of this study was to evaluate the effect of $\mathrm{N}$-acetylglucosamine on the platelets of subjects with resistance to insulin compared with healthy subjects. The platelets $(80,000$ platelets $/ \mathrm{mL})$ of subjects with resistance to insulin were washed and then incubated with $\mathrm{N}$-acetylglucosamine for 10 minutes and marked with the antibodies anti-PAC-1 and anti-CD62P bonded to fluorochromes and $\mathrm{Ca}_{2}+$ by means of a Fluo-3 union. Our results indicate that $\mathrm{N}$-acetylolucosamine inhibits thrombin-induced intraplatelet calcium, the joining of antibodies anti-GPIIb/IIla and anti-P-selectine.

Key words: $\mathrm{N}$-acetylglucosamine, platelet activation

\section{Introducción}

Los principales receptores plaquetarios que participan en la hemostasia, permiten interacciones específicas y funcionales entre proteinas adhesivas. Además, otros receptores plaquetarios están implicados en otras funciones, como inflamación, crecimiento tumoral y metástasis, remodelación tisular o como parte de la inmunidad innata. (Clemetson y Clemetson, 2007) y (Rivera y Navarro, 2009).

Las plaquetas responden a múltiples señales extracelulares que conducen a cambios de forma, las extensiones filopodiales y la exocitosis de gránulos necesarios para la hemostasia. (Brass, 2003). Las plaquetas que son hiperactivas o hipoactivas se consideran un riesgo para la salud, debido a que las plaquetas primeras forman coágulos que pueden causar pérdida de flujo sanguíneo y provocar infarto de miocardio o un 
accidente cerebrovascular; mientras que las plaquetas hipoactivas no se adhieren correctamente a las lesiones y no forman coágulos creando lugares para la pérdida excesiva de sangre. (Mostaza y Packham 1977).

Numerosas proteínas que regulan una amplia gama de funciones celulares han demostrado ser O-GlcNAciladas, incluyendo la alteración de la afinidad de unión de ADN, la promoción de la supervivencia durante el estrés ambiental, y el aumento de la motilidad celular basal y la quimiotaxis. (Wells y Hart, 2003) Las proteinas modificadas por O-GlcNAc incluyen factores de transcripción, componentes de señalización y enzimas metabólicas. Desde su descubrimiento, la importancia de O-GlcNAcilación ha aumentado su participación en la regulación de proteínas celulares (Crawford, 2008) y los resultados han sido cada vez relacionados con varias enfermedades humanas, incluyendo la enfermedad cardiovascular, desórdenes neurodegenerativos, diabetes mellitus y cáncer.

El objetivo de este trabajo fué evaluar el efecto de $\mathrm{N}$-acetilglucosamina sobre los receptores Gpllb/ Illa y P-selectina implicados en la activación plaquetaria de sujetos con resistencia a la insulina. Así como la cuantificación de calcio intraplaquetario.

\section{Materiales y métodos}

Cuantificación de receptores de activación plaquetarios: Gp Illb/llla y P-selectina. Plaquetas lavadas de sujetos con resistencia a la insulina $(80,000$ plaquetas $/ \mathrm{mL})$ se incubaron con 200 mM de GlcNAc durante 10 minutos, se fijaron y lavaron. A continuación, se incubaron con anticuerpos específicos para GPIlb/IIla (PAC 1) y P-selectina (CD62P), acoplados a fluorocromos durante 40 minutos, se eliminó el exceso de anticuerpo. La adquisición y análisis de datos se realizó en un Citómetro de flujo MASCQuant (Miltenyl biotec). La determinación se realizó en 8 sujetos insulino-resistentes y 8 sujetos control.

Cuantificación de calcio intraplaquetario. Se obtuvieron plaquetas lavadas de sujetos con resistencia a la insulina $(80,000$ plaquetas $/ \mathrm{mL})$ y se marcaron con fluo-3 AM acetoxymethyl ester $(4 \mu \mathrm{M})$ a $37^{\circ} \mathrm{C}$ durante 30 minutos, se lavaron con CGS. Se realizaron lecturas de movilización de $\left[\mathrm{Ca}^{2}+\right]$ i con $200 \mathrm{mM}$ de $\mathrm{N}$-acetilglucosamina (GICNAc) utilizando un espectrofluorímetro Perkin-Elmer LS55 y se sometió a un escaneo de la
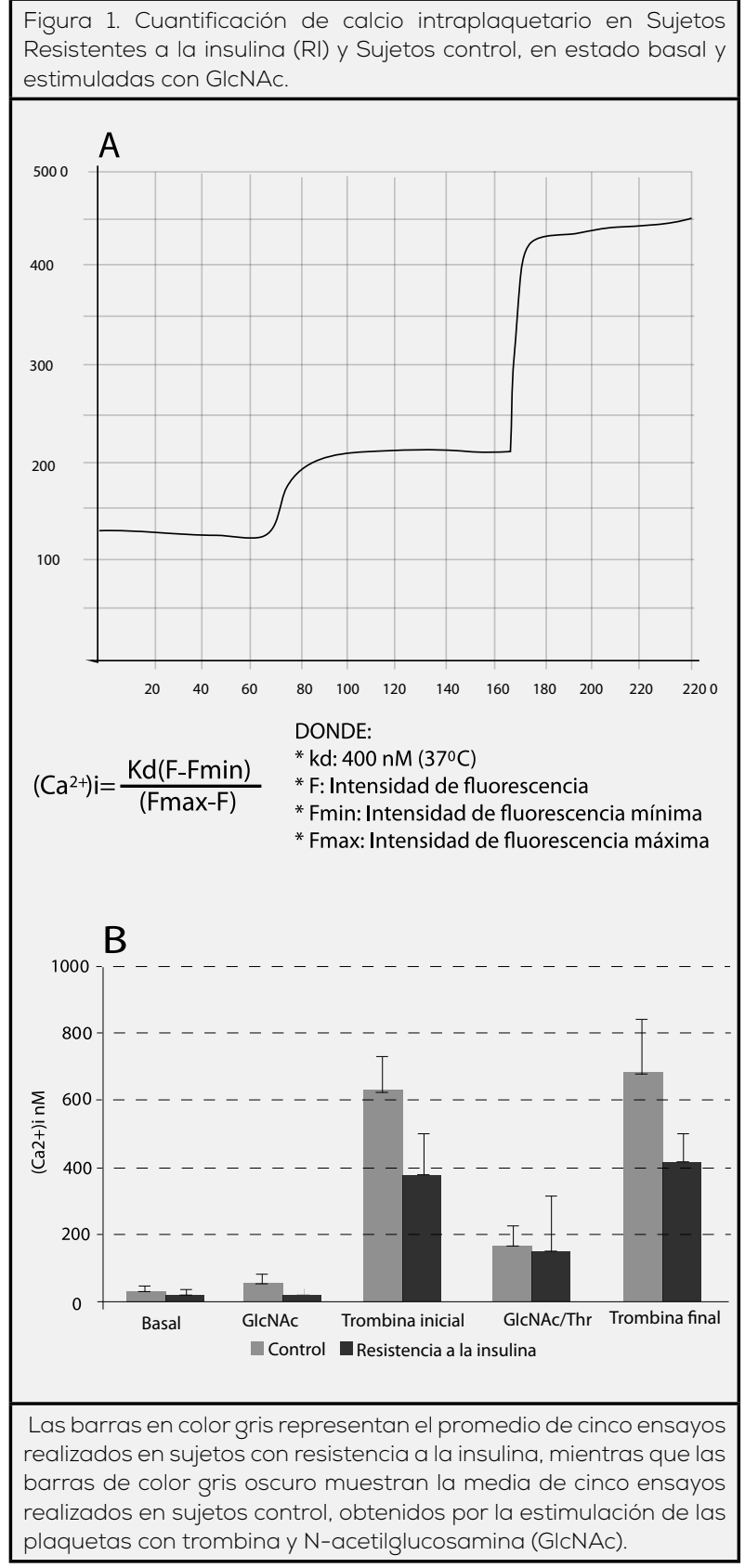

intensidad de fluorescencia contra tiempo (220 segundos), las muestras se excitaron a $505 \mathrm{~nm}$ y la emisión de fluorescencia se recogió a $530 \mathrm{~nm}$. La concentración de Ca2+ intraplaquetario [ $\left.\mathrm{Ca}^{2}+\right]$ i se calculó a partir de las medidas de fluorescencia, empleando la ecuación de Grynkiewicz y colaboradores (figura 1A). Se realiza análisis estadístico: media y desviación estándar, a partir de las concentraciones obtenidas de 5 repeticiones en sujetos control y sujetos con resistencia a la insulina. 


\section{Resultados}

Cuantificación de receptores de activación plaquetarios: Gp Illb/llla y P-selectina. Algunos de los componentes de los gránulos plaquetarios se expresan en las membranas de las plaquetas, como la P-selectina, capaz de permitir la interacción de PSGL-1 con los leucocitos, iniciando así las respuestas inflamatorias y trombóticas. (Vega y Reyes, 2006) y (Graff, 2002). La etapa final es la activación de los receptores $\alpha \operatorname{IIb} \beta 3$, que posibilitan la unión del fibrinógeno y también del FvW. lo que permite el establecimiento de puentes estables entre las plaquetas.(López y Macaya, 2013).

Para evaluar el grado de activación de las plaquetas y el efecto de $\mathrm{N}$-acetilglucosamina, se obtuvieron muestras de 8 sujetos con resistencia a la insulina y 8 sujetos sin resistencia a la insulina. Se determinó la expresión de Gp Ilb/IIla (PAC-1) y de P-selectina (CD62P), en condiciones basales y después de haber sido estimuladas con 200 mM de GlcNAc durante 10 minutos.

Se observó 68\% de expresión de Gp llb/llla en plaquetas de sujetos resistentes a la insulina en estado basal, y cuando son incubadas con GlcNAc, la expresión es cercana a 20\% (figura 2A); mientras que la expresión basal de Gp llb/llla en plaquetas de sujetos sin resistencia a la insulina es de $0.23 \%$ y $19 \%$ cuando las plaquetas son incubadas con GlcNAc (figura 2B). El porcentaje de expresión de P-selectina en sujetos con resistencia a la insulina (figura 2A) es de 10\% y estimuladas con GlcNAc el porcentaje es del 31\%, a diferencia de los sujetos sin resistencia a la insulina; que es del 7\% de expresión basal de P-selectina y $11 \%$ cuando las plaquetas se incubaron con GlcNAc. Se observa un comportamiento diferente de GP llb/ Illa y P-selectina, en ambos tipos de sujetos.

\section{Cuantificación de calcio intraplaquetario.}

Una de las respuestas más tempranas en la fase inicial de la activación plaquetaria, es el aumento en el $\mathrm{Ca++}$ citosólico, que puede ser medido como la alteración de la intensidad de fluorescencia del marcador intracelular ion-sensible Fluo-3 o Indol-1. El calcio liberado por las plaquetas es necesario para la activación de receptores plaquetarios, activación de proteínas y formación de fibrina. (Caen y Wu, 2010).

Se evaluó la concentración de calcio intracelular en las plaquetas de sujetos con y sin resistencia a la insulina, en estado basal e incubado con GlcNAc, figura 1. Se encontró que GlcNAc inhibe la

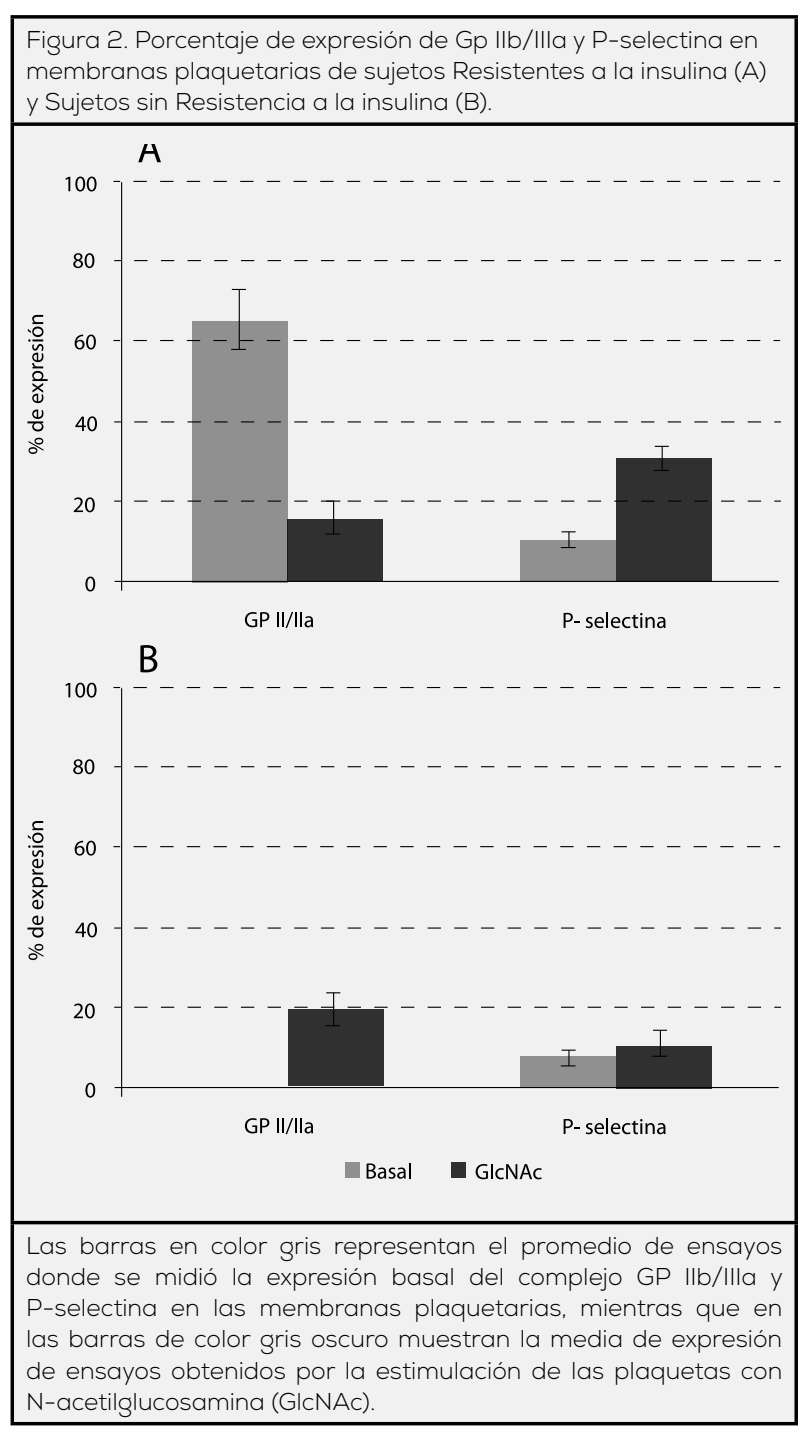

concentración del calcio intracelular liberado por trombina, al observar que a partir de 700 mM como concentración inicial de calcio intraplaquetario inducido por trombina, GlcNAc bajo la concentración a $200 \mathrm{mM}$, en ambos tipos de sujetos.

\section{Discusión}

Las plaquetas estimuladas con GlcNAc inhiben la expresión de GP IIb/IIla y P-selectina que basalmente se encuentran presentes en sujetos con resistencia a la insulina. El aumento de los niveles de fibrinógeno en Diabetes Mellitus parece estar relacionado con inflamación de bajo grado, los niveles de Interleuquina (IL)-6 son elevados y la citoquina es capaz de estimular los hepatocitos para producir fibrinógeno, lo que representa un importante vínculo entre inflamación y el estado de hiper- 
coagulabilidad en Diabetes Mellitus. (Vazzana, et al., 2012). También N-acetilglucosamina es capaz de inducir la translocación de fosfatidilserina en la membrana externa de la plaqueta y provocar su activación.

Se ha asociado que el aumento de la reactividad plaquetaria basal incrementa el riesgo de desarrollar aterotrombosis (Muntaner, et al, 2015). que es una complicación frecuente y letal en pacientes diabéticos, que previamente pudieron haber iniciado solo con resistencia a la insulina.

\section{Discusión}

La activación inducida por la mayoría de los agonistas plaquetarios produce un incremento del $\mathrm{Ca}^{2+}$ libre debido tanto al influjo a través de la membrana, como a la liberación de los depósitos intracelulares, un ejemplo es la liberación de calcio intracelular mediante trombina. Al parecer, $\mathrm{N}$-acetilglucosamina actúa como antagonista al inhibir el calcio intracelular inducido por trombina, aunque utilizado como agonista no parece mostrar algún efecto. Además GlcNAc es capaz de inhibir una alteración que ocurre en la superficie de la membrana durante la activación, es decir, la fusión de las membranas de los gránulos con la membrana plasmática, que puede ser detectada con la expresión de P-selectina y GP IIb/IIla.

Las plaquetas de pacientes con Diabetes Mellitus se caracterizan por la desregulación de varias vías de señalización, que conducen a un fenotipo hiperreactivo con mayor adherencia, activación y agregación. Por lo tanto, las plaquetas responden más a estímulos subumbrales, se consumen más rápidamente lo que resulta en una generación de plaquetas acelerada formando plaquetas hiperreactivas. La hiperglucemia altera la homeostasis del calcio en las plaquetas, aumentado su concentración al facilitar la movilización desde los depósitos intracelulares al citosol, esto sensibiliza la reactividad plaquetaria a los agonistas.

Es importante, por lo tanto, profundizar en el conocimiento de los mecanismos moleculares y celulares involucrados en el proceso de activación plaquetaria, lo cual permitirá desarrollar nuevos biomarcadores terapéuticos.

\section{Referencias bibliográfícas}

Clemetson, K. J., Clemetson, J. M. (2007). "Platelet receptors". En Michelson, A. D., editor. Platelets 2nda ed. Elsevier/Academic Press, San Diego, California. pp.117-43.

Rivera, J., Lozano, M.L., Navarro, L., Vicente, V. (2009) "Platelet receptors and signaling in the dynamics of thrombus formation". En Haematologica, 94, pp.700-711.

Brass, L. "Thrombin and platelet activation" (2003). En Chest Journal, 18S-25S.

Mostaza, J. F., Packham, M. A. (1977) "Platelets and diabetes mellitus". En The New England Journal of Medicine, 297, pp. 1345-1347.

Wells, L., Hart, G. W. (2003) "O-GlcNAc turns twenty: functional implications for post-translational modification of nuclear and cytosolic proteins with a sugar". En FEBS Letter Journal, 546, pp. 154-158.

Crawford, G., et al. (2008) "Murine Platelets are not Regulated by O-Linked $\beta$-Nacetylglucosamine". En Archives Biochemistry Biophysics, June 1, 474(1), pp. 220-224.

Vega, S., Reyes, E., et al. (2006) "Estudio ultraestructural de las plaquetas de pacientes con hemoglobinuria paroxística nocturna". En Revista de Investigación clínica, 58 (5), pp. 475-486.

Graff, et al. (2002) "Close Relationship between the Platelet Activation Marker CD62 and the Granular Release of Platelet-Derived Growth Factor". En The Journal of Pharmacology and Experimental Therapeutics, 300(3), pp. 952-957.

López, F.A., Macaya, C. (2013) "Plaqueta: fisiología de la activación y la inhibición". En Revista Española de Cardiología, 13 (B), pp. 2-7.

Caen, J., Wu, Q. (2010) "Hageman factor, platelets and polyphosphates: early history and recent connection". Journal of Thrombosis and Haemostasis, 8.

Vazzana, N., Ranalli, P., Cuccurullo, Ch., et al. (2012) "Diabetes mellitus and thrombosis". En Thrombosis Research, 129, pp. 371-377.

Muntaner, J., Roggia, R., Badimon, J. (2015) "Diabetes y aterotrombosis. Importante impacto en la carga global de morbilidad. Mecanismos fisiopatológicos involucrados". En Revista Federación Argentina de Cardiología, 44(3), pp. 133-138. 